
\title{
25 Research Square \\ Orbital infarction syndrome in a COVID-19 associated Internal carotid artery occlusion
}

BOBY VARKEY MARAMATTOM ( $\sim$ bobvarkey@gmail.com )

Aster Medcity https://orcid.org/0000-0001-7032-4412

\section{Research Article}

Keywords: COVID-19, orbital infarction syndrome, stroke

Posted Date: July 27th, 2021

DOI: https://doi.org/10.21203/rs.3.rs-601523/v1

License: (c) (i) This work is licensed under a Creative Commons Attribution 4.0 International License. Read Full License 


\section{Abstract}

\section{Main Text}

A 39-year-old man presented with global aphasia and right hemiplegia of 15 hours duration. 12 days earlier, he had developed fever and tested positive for SARS-CoV-2 RT PCR. At admission, he had an NIHSS score of 18. CT brain showed a dense left MCA sign and an area of MCA infarction $<1 / 3 \mathrm{rd}$ of the MCA territory. CT angiogram (CTA) showed a left ICA petro-laceral segment occlusion [large vessel occlusion (LVO)]. MRI showed a partial MCA infarct. He was taken up for mechanical thrombectomy under local anaesthesia. DSA showed an occlusive thrombus in the supraclinoid internal carotid artery (ICA), covering the origin of the left ophthalmic artery. After 5 attempts at aspiration, the anterior cerebral artery and left M1 segments were recanalized with thrombolysis in cerebral infarction (TICl) grade 2a (partial filling $[<2 / 3 r d s]$ ) of the entire vascular territory) revascularization. Final angiographic films showed partial revascularisation of the left ophthalmic artery with very poor orbital collateral flow.

12 hours later, CT showed a completed partial MCA territory infarct. At 24 hours, he was noted to have ptosis of the left eye. Repeat CT brain showed increasing midline shift and he was taken up for decompressive craniectomy. The next day, an ophthalmological evaluation revealed tense left eyelid edema without visible inflammatory signs or orbital bruit. The left pupil was larger than the right and unreactive. There was reduced ocular tonus and conjunctival chemosis. A repeat CT showed enlargement of the muscle bellies of all 4 recti muscles of the left eye which was not seen in the preoperative MRI. The diagnosis of an Orbital infarction syndrome (OIS) with MCA territory stroke due to a left distal ICA occlusion in the setting of COVID-19 was considered.

Orbital infarction in stroke is rare as the orbit is supplied by a rich anastomotic network of vessels. The ophthalmic artery $(O A)$ which is the first branch of the extracavernous ICA often anastomoses with branches from the ECA ensuring that there is adequate blood supply if the ICA, ECA, or the OA are occluded. The most common anastomoses are the posterior (from the middle meningeal artery), the anterior orbit group (from branches of the ECA), and a minor medial group (arteries of the nasal cavity).[1] In a minority of individuals, the $\mathrm{OA}$ is the predominant orbital arterial supplier without any significant collaterals or anastomoses. In such individuals, acute occlusion of the OA can lead to occlusion of both the retinal and the choroidal circulations, (a catastrophic orbital infarction) as in our patient.

After mechanical thrombectomy (MT), an iatrogenic carotid-cavernous fistula [CCF] should be ruled out. A CCF can be distinguished by clinical and radiological features. [Table] MT itself can cause an OIS by producing embolization to new arterial territories in $2 \%$ of patients.[2][3] Aetiologies of an OIS include LVO [ICA or CCA] or dissection, temporal arteritis or other systemic vasculitis, vascular occlusion due to Mucormycosis, embolization of cosmetic fillers or particles during neuro-interventional procedures, head-trauma 
and intracranial surgery.[4],[5] Mucor-mycosis complicating the post-COVID period has been reported to cause OIS.[6]

Acute ischemic stroke occurs in approximately $1.3 \%$ of patients with COVID-19. However, it is associated with higher morbidity and mortality.[7] The unfortunate combination of a prothrombotic state with COVID19 , LVO involving the OA origin, lack of revascularisation of the distal OA and the absence of adequate orbital collateralisation from the ECA branches led to this ICA occlusion producing a catastrophic OIS. Only early revascularisation of the OA can improve the outcome. Most cases are left with residual blindness although anterior ischemia and ophthalmoplegia may resolve with time.

\section{Tables}

Table. Clinico-radiological features of OIS and CCF. ${ }^{[8]}$

\begin{tabular}{|l|l|l|}
\hline Features & Orbital ischemic syndrome & Carotid-cavernous fistula \\
\hline Clinical & Proptosis & Pulsatile proptosis \\
& Blepharoptosis & Orbital bruit \\
& Acute blindness & Chemosis Conjunctival injection \\
& Orbital pain & Subconjunctival hemorrhage \\
& posterior segment ischemia. & Partial ophthalmoplegia \\
& Pale and cyanotic conjunctiva & Arterialization of episcleral veins \\
& Corneal edema & Ocular hypertension (OHT) \\
& Clear lens with a fixed and dilated & Fundoscopy; retinal haemorrhages, \\
& pupil & dilated retinal veins or subtle \\
& Chemosis & optic disc swelling \\
& Ocular hypotony & \\
& Retinal pallor & \\
& Optic disc edema, optic disc pallor & \\
& Severe attenuation of arteries and & \\
& veins & \\
& Diffuse retinal opacification & \\
\hline Radiological & Proptosis & Orbital congestion \\
features & Retrobulbar fat stranding/edema & Proptosis \\
CT/ MRI & Hyperintensity and enlargement & Retrobulbar fat stranding/edema \\
& of extraocular muscles & Enlargement of extraocular muscles \\
& Diffusion restriction in the optic & Venous engorgement \\
& nerve & Enlarged superior ophthalmic vein \\
& & Bulging cavernous sinus \\
& & \\
\hline
\end{tabular}


[1] . Bertelli E, Regoli M, Bracco S. An update on the variations of the orbital blood supply and hemodynamic. Surg Radiol Anat 2017;39:485-96.

[2] . Balami JS, White PM, McMeekin PJ, et al. Complications of endovascular treatment for acute ischemic stroke: prevention and management. Int J Stroke 2018;13:348-61.

[3] . Volders D, Labrie M, Keezer M et al. Exophthalmos following mechanical thrombectomy for anterior circulation stroke: A retrospective study and review of literature. Interv Neuroradiol. 2020;26:416-419.

[4] . Borruat FX, Bogousslavsky J, Uffer S, Klainguti G, Schatz NJ. Orbital infarction syndrome. Ophthalmology. 1993;100:562-8.

[5] . Larrosa Campo D, Fuentes Castañón D, Calleja Puerta S. Global Orbital Infarction Syndrome After a Carotid Artery Dissection. JAMA Neurol. 2019:76:111-112.

[6] . Rao, Raksha; Shetty, Adheesh P; Nagesh, Chinmay $\mathrm{P}^{1}$ Orbital infarction syndrome secondary to rhinoorbital mucormycosis in a case of COVID-19, Indian Journal of Ophthalmology: 2021:69: 1627-1630.

[7] . Qureshi Al, Baskett WI, Huang W et al. Acute Ischemic Stroke and COVID-19: An Analysis of 27676 Patients. Stroke. 2021:52:905-912

[8] . Fargeot G, Lecler A, Lamirel C, Savatovsky J, Obadia M. MRI findings in orbital infarction syndrome. Rev Neurol (Paris). 2018:174:571-573

\section{Declarations}

Patient consent was obtained for the article.

Competing interests: The authors declare no competing interests.

\section{Figures}



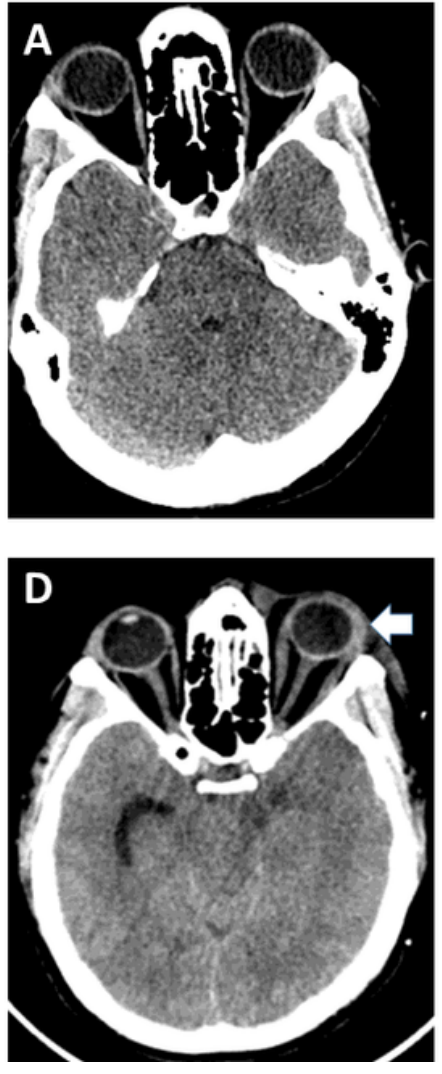
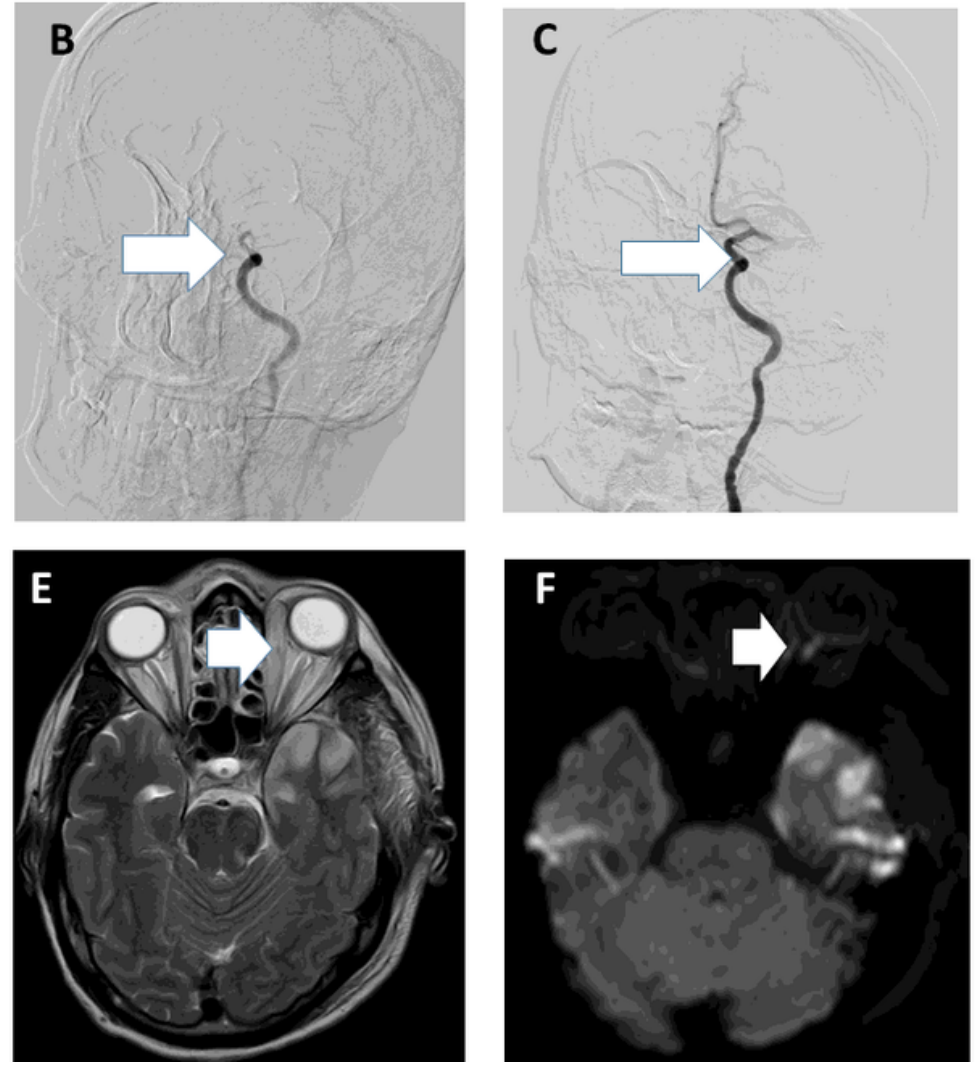

\section{Figure 1}

Panel A. Initial axial CT showing normal orbital muscles. Panel B. DSA showing terminal ICA occlusion. Panel C. Final DSA showing partial recanalization of the left OA (white arrow). Panel D. Axial CT showing enlarged orbital muscles. Panel E. Axial T2 weighted MRI image showing enlarged orbital muscles. Panel F. Axial Diffusion weighted MRI image showing distal optic nerve diffusion restriction. 